\title{
Planar and Nonplanar Solitary Waves in a Four-Component Relativistic Degenerate Dense Plasma
}

\author{
M. R. Hossen, L. Nahar, and A. A. Mamun \\ Department of Physics, Jahangirnagar University, Savar, Dhaka 1342, Bangladesh \\ Correspondence should be addressed to M. R. Hossen; rasel.plasma@gmail.com
}

Received 31 May 2014; Revised 3 September 2014; Accepted 4 September 2014; Published 23 October 2014

Academic Editor: Milan S. Dimitrijevic

Copyright (C) 2014 M. R. Hossen et al. This is an open access article distributed under the Creative Commons Attribution License, which permits unrestricted use, distribution, and reproduction in any medium, provided the original work is properly cited.

\begin{abstract}
The nonlinear propagation of electrostatic perturbation modes in an unmagnetized, collisionless, relativistic, degenerate plasma (containing both nonrelativistic and ultrarelativistic degenerate electrons, nonrelativistic degenerate ions, and arbitrarily charged static heavy ions) has been investigated theoretically. The Korteweg-de Vries (K-dV) equation has been derived by employing the reductive perturbation method. Their solitary wave solution is obtained and numerically analyzed in case of both planar and nonplanar (cylindrical and spherical) geometry. It has been observed that the ion-acoustic (IA) and modified ion-acoustic (mIA) solitary waves have been significantly changed due to the effects of degenerate plasma pressure and number densities of the arbitrarily charged heavy ions. It has been also found that properties of planar K-dV solitons are quite different from those of nonplanar K-dV solitons. There are numerous variations in case of mIA solitary waves due to the polarity of heavy ions. The basic features and the underlying physics of IA and mIA solitary waves, which are relevant to some astrophysical compact objects, are briefly discussed.
\end{abstract}

\section{Introduction}

A large fraction of matter in the universe is in the plasma state. Significant attention has been devoted to the study of ion-acoustic (IA) waves in plasmas not only from an academic point of view, but also from the view of its vital role in understanding the nonlinear features of localized electrostatic disturbances in laboratory and space environments [1-6]. The basic features of solitary waves associated with IA waves, in which electron thermal pressure gives rise to a restoring force and ion mass provides the inertia, were first theoretically predicted by Washimi and Tanuiti by assuming an ideal plasma containing cold ions and isothermal electrons. These basic features [7] were verified by a novel laboratory experiment of Ikezi et al. [6].

Presently, relativistic degeneracy of plasmas has received great attention because of its vital role in different astrophysical environments $[8,9]$, where particle velocities become comparable to the speed of light. Astrophysical compact objects such as white dwarfs, neutron stars, quasars, black holes, and pulsars are examples where relativistic degenerate plasmas are dominant and interesting new phenomena are investigated by several nonlinear effects in such plasmas. The basic constituents of white dwarfs are mainly oxygen, carbon, and helium with an envelope of hydrogen gas. In some relatively massive white dwarfs, one can think of the presence of heavier element like iron within the stars. The existence of heavy elements is found to form in a prestellar stage of the evolution of the universe, when all matter was compressed to extremely high densities and possessed correspondingly high temperatures [10]. In case of such a compact object the degenerate electron number density is so high (in white dwarfs it can be of the order of $10^{30} \mathrm{~cm}^{-3}$, even more [8]).

Chandrasekhar [11, 12] presented a general expression for the relativistic ion and electron pressures in his classical papers. The pressure for ion fluid can be given by the following equation:

$$
P_{i}=K_{i} n_{i}^{\alpha},
$$

where

$$
\alpha=\frac{5}{3} ; \quad K_{i}=\frac{3}{5}\left(\frac{\pi}{3}\right)^{1 / 3} \frac{\pi \hbar^{2}}{m} \simeq \frac{3}{5} \Lambda_{c} \hbar c,
$$


for the nonrelativistic limit (where $\Lambda_{c}=\pi \hbar / m c=1.2 \times$ $10^{-10} \mathrm{~cm}$ and $\hbar$ is the Planck constant divided by $2 \pi$ ), while for the electron fluid,

$$
P_{e}=K_{e} n_{e}^{\gamma}
$$

where

$$
\begin{gathered}
\gamma=\alpha ; \quad K_{e}=K_{i} \quad \text { for nonrelativistic limit, } \\
\gamma=\frac{4}{3} ; \quad K_{e}=\frac{3}{4}\left(\frac{\pi^{2}}{9}\right)^{1 / 3} \hbar c \simeq \frac{3}{4} \hbar c,
\end{gathered}
$$

in the ultrarelativistic limit [11-14].

We note that we have considered both electron and ion degeneracy, but light electrons are assumed to be relativistic (nonrelativistic and ultrarelativistic) and ions (which are heavier than electrons) are assumed to be nonrelativistic. Equations (1) and (3) represent the equations of state of ion and electron pressure in high density regime.

Recently, several authors have used the pressure laws to observe the linear and nonlinear properties of electrostatic [15-19] and electromagnetic [20, 21] waves by using only nonrelativistic or both nonrelativistic and ultrarelativistic quantum hydrodynamics (QHD) [22] and quantummagnetohydrodynamics (QMHD) models $[23,24]$. The solution of the Dirac equation for electrostatic and electromagnetic waves in a relativistic quantum plasmas has been discussed by Mendonca and Serbeto [25]. Masood and Eliasson considered an unmagnetized quantum plasma with relativistically degenerate electrons and cold fluid ions and they studied the basic features of the solitary structures [26]. Later on, Shukla et al. [27] theoretically investigated the nonlinear propagation of electrostatic waves in degenerate quantum plasma. They considered strongly coupled nondegenerate ions and degenerate electron fluids in an unmagnetized dense plasma and studied the basic properties of solitary and shock structures. Zeba et al. [28] considered a warm collisionless electron-positron-ion plasma with ultrarelativistic degenerate electrons and positrons and investigated theoretically the existence regions for ion solitary pulses. Since the dense astrophysical quantum plasmas can be confined by stationary heavy ions, therefore the effect of the heavy ions has to be taken into account, especially for astrophysical observations (such as white dwarfs, neutron stars, and black holes) where the degenerate plasma pressure and heavy ions play an important role in the formation and stability of the existing waves. Recently, Zobaer et al. $[9,15,16,29]$ also considered nonrelativistic ion fluids and both nonrelativistic and ultrarelativistic electron fluids and theoretically investigated the basic features of solitary, shock, and double layer structures. All of these investigations are mainly based on planar geometry and they did not consider the effect of heavy ions, which may not be a realistic situation in space environments. Since the waves observed in astrophysical compact objects are certainly not infinite in one dimension (1D) and there is a great possibility of having both positively and negatively charged heavy ions, there are several cases of practical importance where planar geometry does not work and one would have to consider a nonplanar geometry. The notable examples where nonplanar geometry plays a vital role are white dwarfs, neutron stars, black holes, circumstellar disks, dark molecular clouds, cometary tails, and so forth. Till now, no theoretical investigation has been made to study the extreme conditions of matter for both nonrelativistic and ultrarelativistic limits and arbitrarily (both positively and negatively) charged heavy ions on a planar and nonplanar (cylindrical and spherical) geometry. Therefore, in our present work, we attempt to study the basic features of planar and nonplanar IA and mIA solitary waves by deriving the Korteweg-de Vries equation in a dense plasma containing degenerate electron and ion fluids and arbitrarily charged static heavy ions.

The paper is organized as follows. The governing equations are provided in Section 2. The K-dV equation is derived in Section 3. The profiles of $\mathrm{K}-\mathrm{dV}$ solitons are presented in Section 4 and numerically analyzed in order. Finally, a brief discussion is given in Section 5.

\section{Governing Equations}

We consider a nonplanar (cylindrical and spherical) geometry and nonlinear propagation of mIA waves (consisting of arbitrarily charged static heavy ions, nonrelativistic degenerate cold ions, and both nonrelativistic and ultrarelativistic degenerate electron fluids) in an unmagnetized, collisionless dense plasma. Hence, at equilibrium, we have $Z_{i} n_{i 0}+j Z_{h} n_{h 0}=$ $n_{e 0}$, where $n_{s 0}$ is the particle number density of the species $s$ with $s=e(i) h$ for electrons (ions) heavy ions, $Z_{h}$ is the number of ions residing onto the heavy ion surface, and $e$ is the magnitude of charge of an electron and $j=+1(j=-1)$ for positively (negatively) charged heavy ions. The nonlinear dynamics of the electrostatic waves propagating in such a degenerate dense plasma system is governed by the following normalized equations:

$$
\begin{aligned}
& \frac{\partial n_{i}}{\partial t}+\frac{1}{r^{\nu}} \frac{\partial}{\partial r}\left(r^{\nu} n_{i} u_{i}\right)=0 \\
& \frac{\partial u_{i}}{\partial t}+u_{i} \frac{\partial u_{i}}{\partial r}+\frac{\partial \phi}{\partial r}+\frac{K_{1}}{n_{i}} \frac{\partial n_{i}^{\alpha}}{\partial r}=0, \\
& n_{e} \frac{\partial \phi}{\partial r}-K_{2} \frac{\partial n_{e}^{\gamma}}{\partial r}=0, \\
& \frac{1}{r^{\nu}} \frac{\partial}{\partial r}\left(r^{\nu} \frac{\partial \phi}{\partial r}\right)=-\rho, \\
& \rho=n_{i}-(1+j \mu) n_{e}+j \mu,
\end{aligned}
$$

where $v=0$ for one-dimensional planar geometry and $\nu=1$ (2) for nonplanar cylindrical (spherical) geometry; $n_{i}$ $\left(n_{e}\right)$ is the ion (electron) number densities normalized by its equilibrium value $n_{i 0}\left(n_{e 0}\right), u_{i}$ is the ion fluid speed normalized by $C_{i}=\left(m_{e} c^{2} / m_{i}\right)^{1 / 2}$ with $m_{e}\left(m_{i}\right)$ being the electron (ion) rest mass, $c$ is the speed of light in vacuum, and $\phi$ is the electrostatic wave potential normalized by $m_{e} c^{2} / e$ with $e$ being the magnitude of the charge of an electron. Here $\mu(=$ $\left.Z_{h} n_{h 0} / Z_{p} n_{i 0}\right)$ is the ratio of the number density of heavy ions and positive ions multiplied by their charge per ion $\left(Z_{h} / Z_{p}\right)$. 
When $\mu=0$ the situation acts as normal electron-ion plasma system. The polarity of the heavy ions depends on the parameter $j$. When $j=+1(-1)$ the heavy ions act as positively (negatively) charged in this plasma system. The time variable $(t)$ is normalized by $\omega_{p i}=\left(4 \pi n_{0} e^{2} / m_{i}\right)^{1 / 2}$, and the space variable $(r)$ is normalized by $\lambda_{s}=\left(m_{e} c^{2} / 4 \pi n_{0} e^{2}\right)^{1 / 2}$. The relativistic constants are $K_{1}=n_{i_{0}}^{\alpha-1} K_{i} / m_{e} c^{2}, K_{2}=$ $n_{e_{0}}^{\gamma-1} K_{e} / m_{e} c^{2}, K_{1}^{\prime}=\alpha K_{1}$, and $K_{2}^{\prime}=\gamma K_{2}$.

\section{Derivation of K-dV Equation}

Now we derive the K-dV equation by employing the reductive perturbation technique to examine the electrostatic perturbations propagating in this dense plasma system and introduce the stretched coordinates [30] as follows:

$$
\begin{gathered}
\xi=-\epsilon^{1 / 2}\left(r+V_{p} t\right), \\
\tau=\epsilon^{3 / 2} t,
\end{gathered}
$$

where $V_{p}$ is the wave phase speed $(\omega / k$ with $\omega$ being the angular frequency and $k$ being the wave number of the perturbation mode) and $\epsilon$ is a smallness parameter measuring the weakness of the dispersion $(0<\epsilon<1)$. We then expand the parameters $n_{i}, n_{e}, u_{i}, \phi$, and $\rho$ in power series of $\epsilon$ :

$$
\begin{gathered}
n_{i}=1+\epsilon n_{i}^{(1)}+\epsilon^{2} n_{i}^{(2)}+\cdots, \\
n_{e}=1+\epsilon n_{e}^{(1)}+\epsilon^{2} n_{e}^{(2)}+\cdots, \\
u_{i}=\epsilon u_{i}^{(1)}+\epsilon^{2} u_{i}^{(2)}+\cdots, \\
\phi=\epsilon \phi^{(1)}+\epsilon^{2} \phi^{(2)}+\cdots, \\
\rho=\epsilon \rho^{(1)}+\epsilon^{2} \rho^{(2)}+\cdots .
\end{gathered}
$$

Now, expressing (5) (using (6)) in terms of $\xi$ and $\tau$ and substituting (7) into (5), one can easily develop different sets of equations in various powers of $\epsilon$. To the lowest order in $\epsilon$, we have $u_{i}^{(1)}=-V_{p} \phi^{(1)} /\left(V_{p}^{2}-K_{1}^{\prime}\right), n_{i}^{(1)}=\phi^{(1)} /\left(V_{p}^{2}-K_{1}^{\prime}\right)$, $n_{e}^{(1)}=\phi^{(1)} / K_{2}^{\prime}$, and $V_{p}=\sqrt{\left(K_{2}^{\prime} /(1+j \mu)\right)+K_{1}^{\prime}}$, where $K_{1}^{\prime}=$ $\alpha K_{1}$ and $K_{2}^{\prime}=\gamma K_{2}$. The relation $V_{p}=\sqrt{\left(K_{2}^{\prime} /(1+j \mu)\right)+K_{1}^{\prime}}$ represents the dispersion relation as well as the phase velocity for the mIA-type electrostatic waves in this degenerate plasma under consideration.

To the next higher order in $\epsilon$, we obtain a set of equations:

$$
\begin{gathered}
\frac{\partial n_{i}^{(1)}}{\partial \tau}-V_{p} \frac{\partial n_{i}^{(2)}}{\partial \xi}-\frac{\partial}{\partial \xi}\left[u_{i}^{(2)}+n_{i}^{(1)} u_{i}^{(1)}\right]-\frac{v u_{i}^{(1)}}{V_{p} \tau}=0 \\
\frac{\partial u_{i}^{(1)}}{\partial \tau}-V_{p} \frac{\partial u_{i}^{(2)}}{\partial \xi}-u_{i}^{(1)} \frac{\partial u_{i}^{(1)}}{\partial \xi}-\frac{\partial \phi^{(2)}}{\partial \xi} \\
-K_{1}^{\prime} \frac{\partial}{\partial \xi}\left[n_{i}^{(2)}+\frac{(\alpha-2)}{2}\left(n_{i}^{(1)}\right)^{2}\right]=0
\end{gathered}
$$

$$
\begin{gathered}
\frac{\partial \phi^{(2)}}{\partial \xi}-K_{2}^{\prime} \frac{\partial}{\partial \xi}\left[n_{e}^{(2)}+\frac{(\gamma-2)}{2}\left(n_{e}^{(1)}\right)^{2}\right]=0, \\
\frac{\partial^{2} \phi^{(1)}}{\partial \xi^{2}}=-\rho^{(1)}, \\
\rho=n_{i}-(1+j \mu) n_{e}+j \mu .
\end{gathered}
$$

Now, combining (8) we deduce a Korteweg-de Vries (K-dV) equation:

$$
\frac{\partial \phi^{(1)}}{\partial \tau}+A \phi^{(1)} \frac{\partial \phi^{(1)}}{\partial \xi}+B \frac{\partial^{3} \phi^{(1)}}{\partial \xi^{3}}+\frac{\nu \phi^{(1)}}{2 \tau}=0,
$$

where

$$
\begin{aligned}
& A=\frac{\left(V_{p}^{2}-K_{1}^{\prime}\right)^{2}}{2 V_{p}}\left[\frac{3 V_{p}^{2}+K_{1}^{\prime}(\alpha-2)}{\left(V_{p}^{2}-K_{1}^{\prime}\right)^{3}}+\frac{(\gamma-2)(1+j \mu)}{K_{2}^{\prime 2}}\right], \\
& B=\frac{\left(V_{p}^{2}-K_{1}^{\prime}\right)^{2}}{2 V_{p}} .
\end{aligned}
$$

\section{Numerical Analysis and Results}

Generally, IA waves are low frequency electrostatic waves in which inertia is provided by ion mass and restoring force comes from electron thermal pressure. The modified ionacoustic (mIA) waves are nothing but IA-type waves in the presence of static heavy ions. It is important to note that if we neglect the heavy ions then we get the usual IA waves. In order to analyze the IA and mIA solitary waves, we turn to (9) with the term $\nu \phi^{(1)} / 2 \tau$, which is due to the effects of the nonplanar (cylindrical and spherical) geometry. An exact analytic solution of (9) is not possible. However, for clear understanding, we first briefly discuss the stationary solitary wave solution for (9) with $v=0$, though the solution is similar for both IA and mIA waves. We should note that for large value of $\tau$, the term $\nu \phi^{(1)} / 2 \tau$ is negligible. So, in our numerical analysis, we start with a large value of $\tau$ (namely, $\tau=-14$ ), and at this large (negative) value of $\tau$, we choose the stationary solitary wave solution of (9) (without the term $\nu \phi^{(1)} / 2 \tau$ ) as our initial pulse. For a moving frame moving with a speed $u_{0}$, the stationary solitary wave solution of (9) is given by

$$
\phi_{(\nu=0)}^{(1)}=\phi_{m} \operatorname{sech}^{2}\left(\frac{\xi}{\Delta}\right)
$$

where the maximum amplitude $\phi_{m}=3 u_{0} / A$, and the width

$$
\Delta=\left(\frac{4 B}{u_{0}}\right)^{1 / 2} \text {. }
$$

The profiles of solitary waves caused by the balance between nonlinearity and dispersion are shown in Figures 3 to 14 , which show how the effects of cylindrical $(\nu=1)$ and 
spherical $(\nu=2)$ geometry modify the time-dependent IA and mIA solitary structures and how it differs from planar $(\nu=0)$ ones.

The conditions for the existence of cylindrical and spherical solitary structures and their basic features are found to be significantly modified in the presence of nonrelativistic ions, both nonrelativistic and ultrarelativistic electrons and arbitrarily charged static heavy ions. The IA and mIA waves are modified when ion is nonrelativistic degenerate $(\alpha=5 / 3)$ and electron is ultrarelativistic degenerate $(\gamma=4 / 3)$ than both electron and ion being nonrelativistic degenerate $(\alpha=$ $\gamma=5 / 3)$. The ranges $\left(u_{0}=0.01-1\right.$ and $\left.\mu=0.2-0.6\right)$ [1315 ] of plasma parameters used in this numerical analysis are very wide and correspond to space and laboratory plasma situations.

The nonlinearity has an important role as the solitary waves are caused by the balance between nonlinearity and dispersion. This is a unique phenomenon that the degree of nonlinearity is proportional to the potential of the plasma system as a highly nonlinear medium causes high electrostatic potential. We have found that the phase speed with negatively (positively) charged heavy ions for ultrarelativistic case is higher (lower) than that for nonrelativistic case (it is expected from the expression of $V_{p}$ ). We have also found that as time decreases the amplitude of the solitary waves in cylindrical and spherical geometry increases. It is also examined that, in spherical geometries, the amplitude is always distinctly higher than cylindrical geometries for K-dV solitons, which indicates that the density compression can be more effectively obtained in a spherical geometry. It is observed that the amplitude of the K-dV solitons is always distinctly higher for nonrelativistic case than for ultrarelativistic one. It is due to the effects of relativistic factors; that is, $\alpha>\gamma$ for ultrarelativistic case and $\alpha=\gamma$ for nonrelativistic case. We also found that the amplitude of the K-dV solitons is lower for positively charged heavy ions than for negatively charged heavy ions. Actually, this happens due to the reason that it decreases the value of the nonlinearity coefficient $A$. It is obvious from (12) that the height of the amplitude of the solitary structures is directly proportional to the solitary speed moving with speed $u_{0}$ and inversely proportional to $A$. On the other hand, (12) also implies that the width of these solitary structures is directly proportional to the square root of the constant $B$ and inversely proportional to the solitary speed moving with speed $u_{0}$.

We have first graphically represented the effects of $\mu$ on the phase speed of mIA waves as shown in Figures 1 and 2. From the figures, for negatively charged static heavy ions $(j=-1)$, we observe that the upper (dashed orange) line represents the ultrarelativistic case and the lower (dashed blue) line represents the nonrelativistic case. On the other hand, for positively charged static heavy ions $(j=+1)$, the upper (dashed red) line represents the nonrelativistic case and the lower (dashed green) line represents the ultrarelativistic case. The profiles of IA and mIA waves for 1D planar $(\nu=0)$ system comparing the nonrelativistic and ultrarelativistic limits are shown in Figures 3 and 4.

The effects of degenerate electron and ion fluids and arbitrarily charged static heavy ions significantly modify

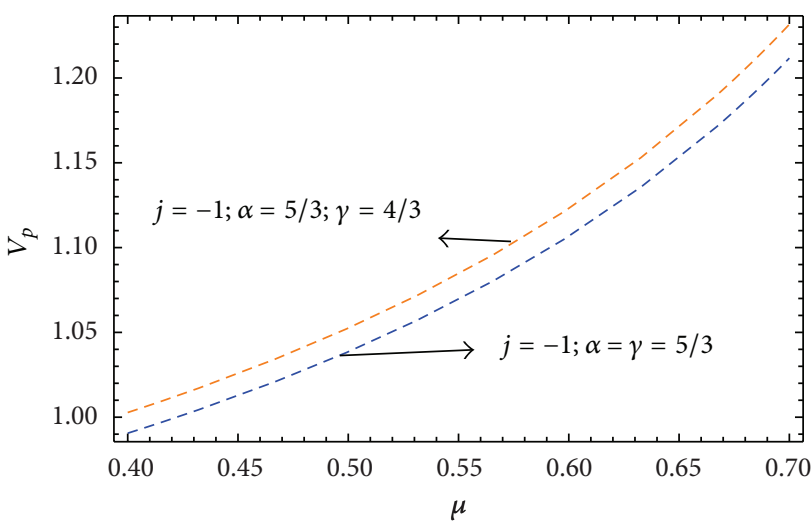

FIgURE 1: Showing the effects of $\mu$ on the phase speed of mIA $\mathrm{K}-\mathrm{dV}$ solitons for negatively charged static heavy ions. The upper (dashed orange) line represents the ultrarelativistic case and the lower (dashed blue) line the nonrelativistic case.

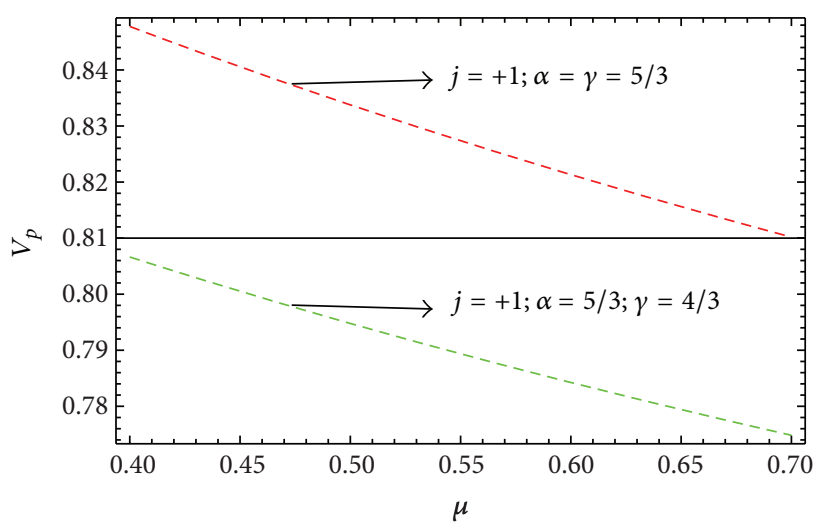

FIGURE 2: Showing the effects of $\mu$ on the phase speed of mIA K-dV solitons for positively charged static heavy ions. The upper (dashed red) line represents the nonrelativistic case and the lower (dashed green) line the ultrarelativistic case.



FIGURE 3: Showing the variation of amplitude of IA K-dV solitons in case of planar $(\nu=0)$ geometry. The lower (purple) line represents ion being nonrelativistic degenerate and electron being ultrarelativistic degenerate and the upper (red) one represents both electron and ion being nonrelativistic degenerate. 


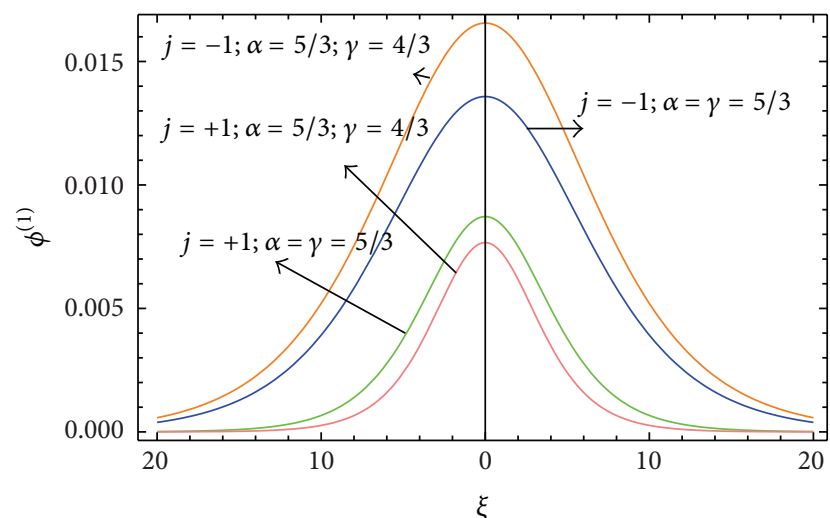

FIGURE 4: Showing the variation of amplitude of mIA K-dV solitons in the presence of arbitrarily charged static heavy ions for planar $(\nu=0)$ geometry. The upper orange and blue lines represent the relativistic cases for negatively charged static heavy ions and the lower green and pink lines represent the relativistic cases for positively charged static heavy ions.

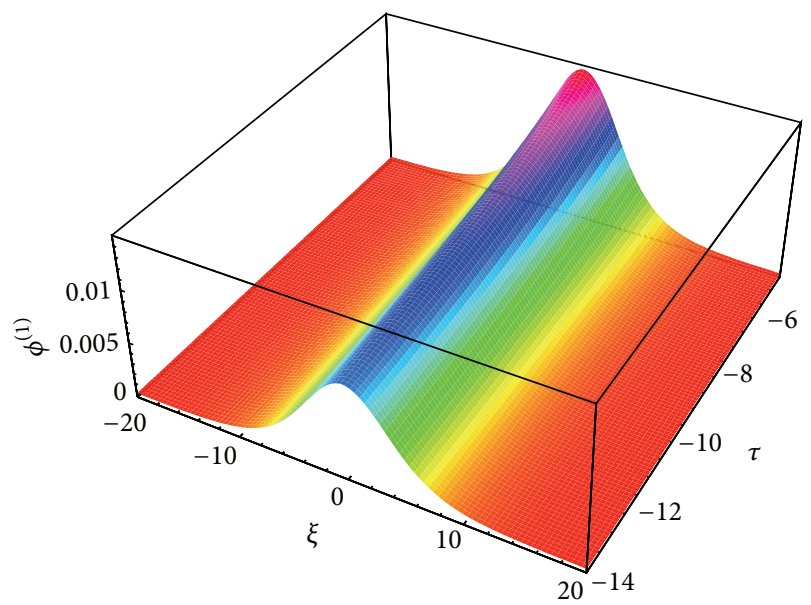

FIGURE 5: Effects of cylindrical geometry on mIA K-dV solitons in the presence of positively charged static heavy ions when both electron and ion are nonrelativistic degenerate $(\nu=1 ; j=+1$; $\left.\mu=0.5 ; n_{e 0}=1.1 \times 10^{30} ; u_{0}=0.01 ; \alpha=\gamma=5 / 3\right)$.

the basic properties (speed, amplitude, and width) of the IA and mIA K-dV solitons. When we compare the $v=0$ case for IA wave (Figure 3), we observe that the red line represents the nonrelativistic case and the purple line represents the ultrarelativistic case. But in case of mIA wave, for negatively charged heavy ions $(j=-1)$, we found that the upper orange line represents the ultrarelativistic case and the lower blue line represents the nonrelativistic case (Figure 4). On the other hand, for positively charged heavy ions $(j=+1)$, the upper green line represents the nonrelativistic case and the lower pink line represents the ultrarelativistic case (Figure 4). After that, we observe the $v=1$ and $v=2$ case for both IA (Figures 13-14) and mIA waves (Figures 5-12) comparing the nonrelativistic and ultrarelativistic limits. Finally, the results that we have found in this investigation can be summarized as follows.

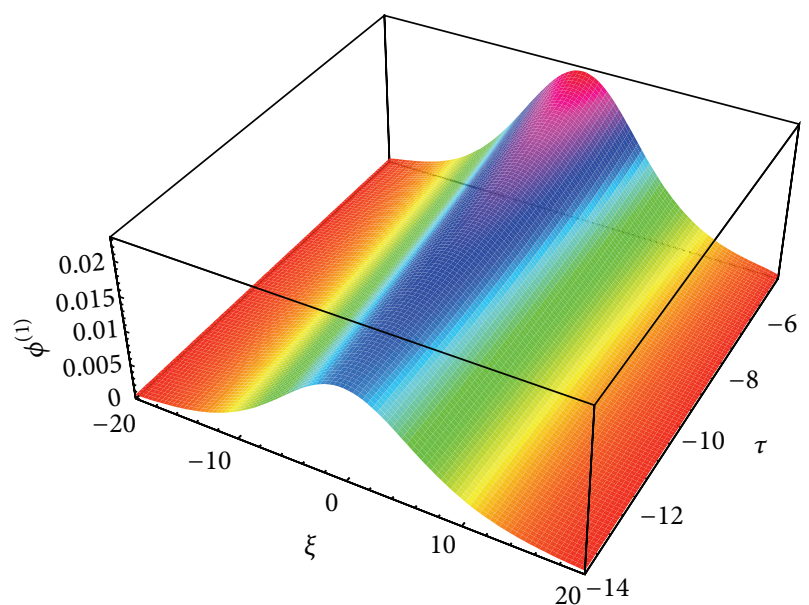

FIGURE 6: Effects of cylindrical geometry on mIA K-dV solitons in the presence of negatively charged static heavy ions when both electron and ion are nonrelativistic degenerate $(\nu=1 ; j=-1$; $\left.\mu=0.5 ; n_{e 0}=1.1 \times 10^{30} ; u_{0}=0.01 ; \alpha=\gamma=5 / 3\right)$.

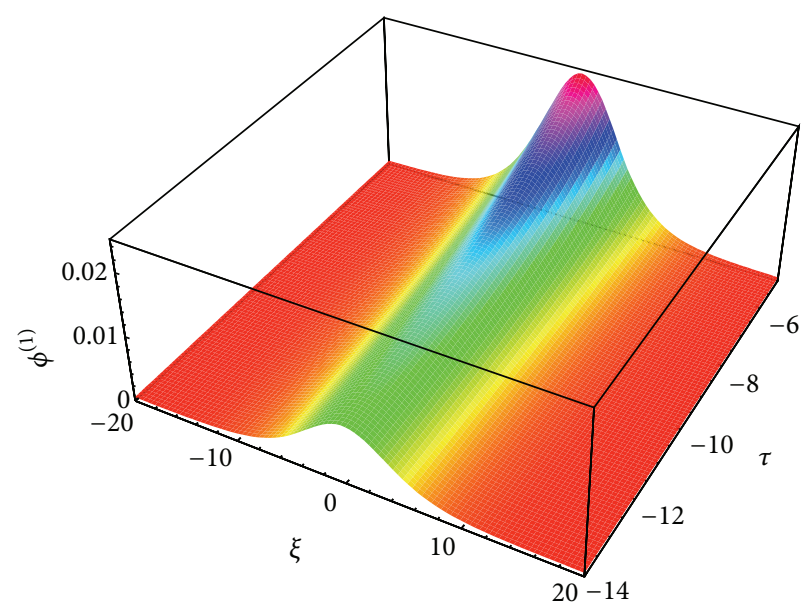

FIGURE 7: Effects of spherical geometry on mIA K-dV solitons in the presence of positively charged static heavy ions when both electron and ion are nonrelativistic degenerate $\left(\nu=2 ; j=+1 ; \mu=0.5 ; n_{e 0}=\right.$ $\left.1.1 \times 10^{30} ; u_{0}=0.01 ; \alpha=\gamma=5 / 3\right)$.

(1) The plasma system under consideration supports finite amplitude K-dV solitons, whose basic properties (polarity, amplitude, width, etc.) depend on the degenerate electrons and the arbitrarily charged stationary heavy ions number densities.

(2) For negatively (positively) charged heavy ions, the phase speed increases (decreases) with the increase of $\mu$ (Figures 1 and 2). The phase speed is always higher (lower) for negatively (positively) charged static heavy ions for ultrarelativistic case than for nonrelativistic case.

(3) We can compare the IA and mIA solitary structures in case of planar geometry as shown in Figures 3 and 4. In these profiles, we observe that the amplitude is higher (lower) for negatively (positively) charged 


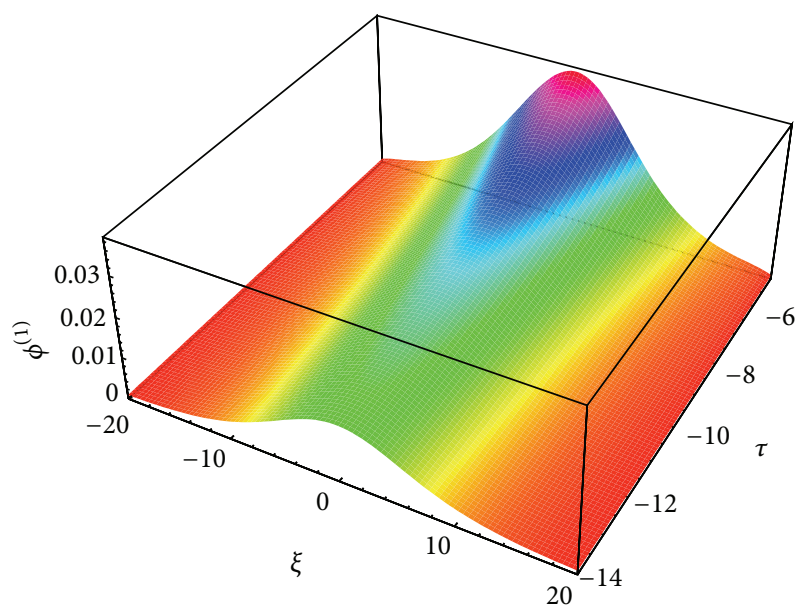

FIGURE 8: Effects of spherical geometry on mIA K-dV solitons in the presence of negatively charged static heavy ions when both electron and ion are nonrelativistic degenerate $\left(\nu=2 ; j=-1 ; \mu=0.5 ; n_{e 0}=\right.$ $1.1 \times 10^{30} ; u_{0}=0.01 ; \alpha=\gamma=5 / 3$ ).

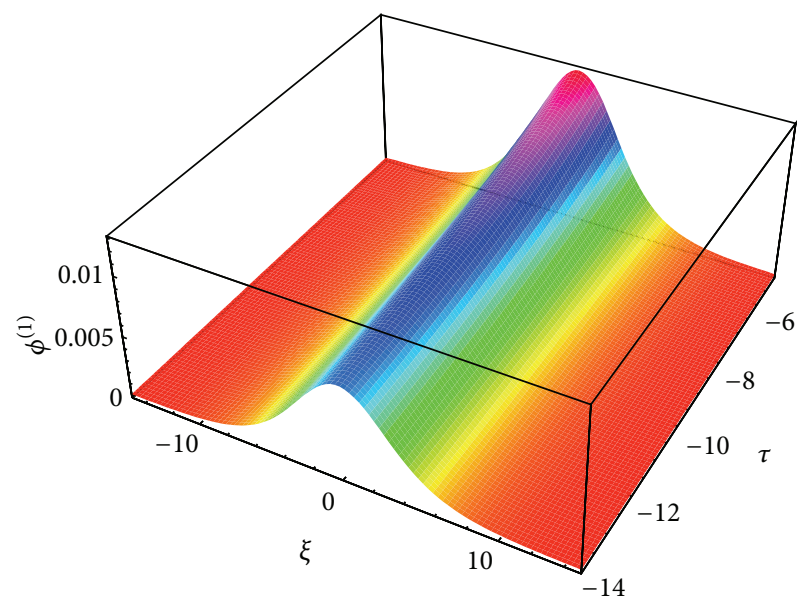

FIGURE 9: Effects of cylindrical geometry on mIA K-dV solitons in the presence of positively charged static heavy ions when ion is nonrelativistic degenerate and electron is ultrarelativistic degenerate $\left(\nu=1 ; j=+1 ; \mu=0.5 ; n_{e 0}=1.1 \times 10^{30} ; u_{0}=0.01 ; \alpha=5 / 3 ; \gamma=4 / 3\right)$.

mIA waves than for the IA ones. It is also valid for cylindrical and spherical IA (Figures 13-14) and mIA (Figures 5-12) solitary structures.

(4) The large value of $\tau$ kills the possibility of formation of nonplanar solitons. It has been found that as the value of $\tau$ decreases the amplitude of these localized pulses increases for both IA (Figures 13-14) and mIA (Figures 5-12) waves. From the observation we can say that the amplitude of cylindrical (Figures 13 and 14 for IA and Figures 5, 6, 9, and 10 for mIA wave) $\mathrm{K}-\mathrm{dV}$ solitons is larger than 1D planar (Figure 3 for IA and Figure 4 for mIA wave) ones but smaller than that of the spherical ones (Figures 7, 8, 11, and 12 for mIA wave).

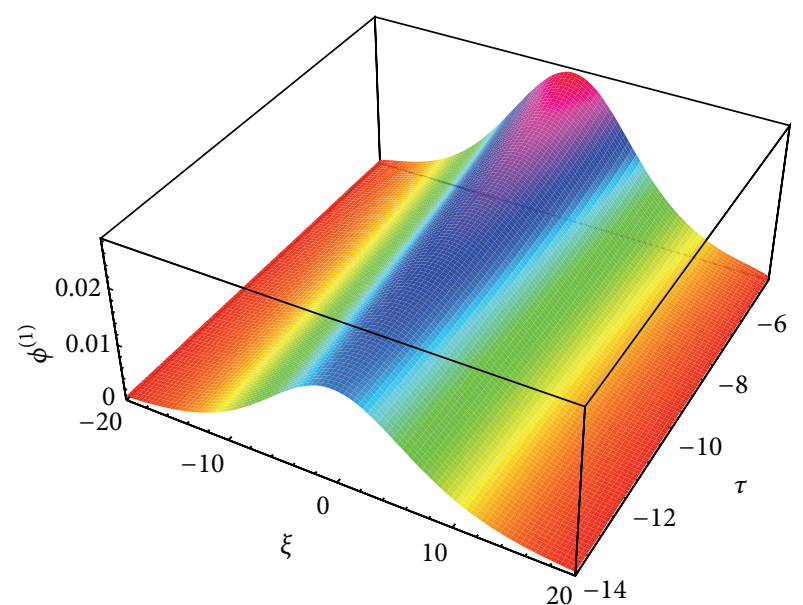

FIGURE 10: Effects of cylindrical geometry on mIA K-dV solitons in the presence of negatively charged static heavy ions when ion is nonrelativistic degenerate and electron is ultrarelativistic degenerate $\left(\nu=1 ; j=-1 ; \mu=0.5 ; n_{e 0}=1.1 \times 10^{30} ; u_{0}=0.01 ; \alpha=5 / 3 ; \gamma=4 / 3\right)$.

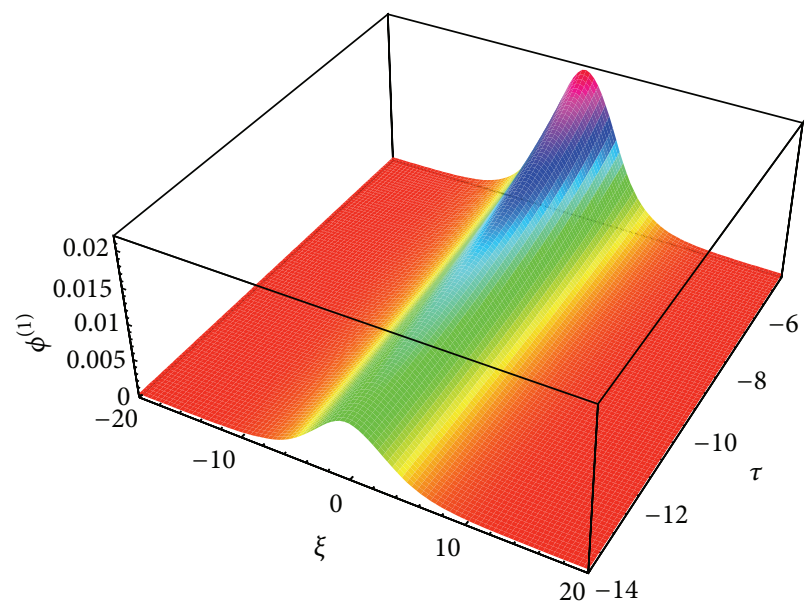

FIGURE 11: Effects of spherical geometry on mIA K-dV solitons in the presence of positively charged static heavy ions when ion is nonrelativistic degenerate and electron is ultrarelativistic degenerate $\left(\nu=2 ; j=+1 ; u_{0}=0.01 ; n_{e 0}=1.1 \times 10^{30} ; \alpha=5 / 3 ; \gamma=4 / 3\right)$.

(5) From the observation of Figure 3, we can clearly say that the amplitude is lower for ultrarelativistic case than for nonrelativistic case and from Figure 4, the amplitude is higher for ultrarelativistic case than for nonrelativistic case. It is also valid for all other cylindrical and spherical graphs of IA (Figures 13-14) and mIA (Figures 5-12) waves.

(6) The amplitude of the mIA K-dV solitons significantly differ with the polarity of heavy ions and relativistic parameters. It is found from Figure 4 that the amplitude of mIA wave in planar case is always higher (lower) for negatively (positively) charged heavy ions for both nonrelativistic and ultrarelativistic limits which is also valid in nonplanar case (Figures 5-12). 


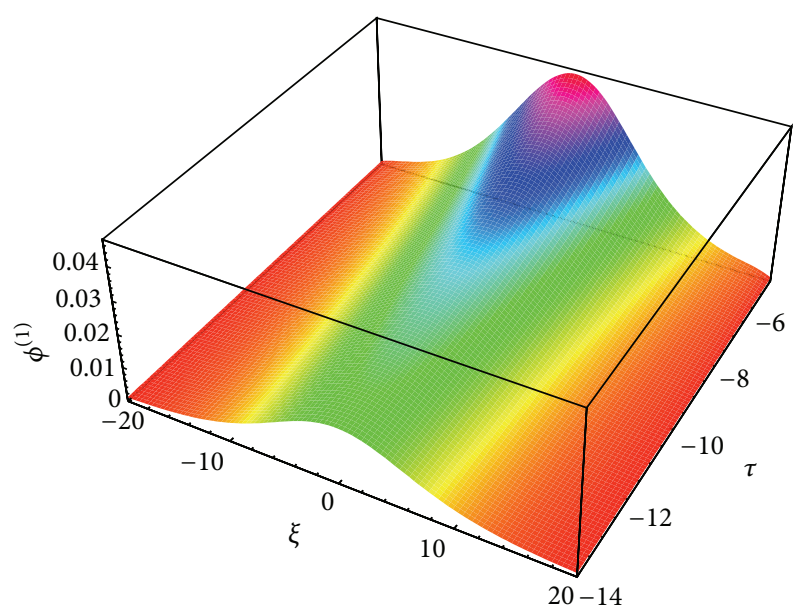

FIGURE 12: Effects of spherical geometry on mIA K-dV solitons in the presence of negatively charged static heavy ions when both electron and ion are nonrelativistic degenerate $(\nu=2 ; j=-1$; $\left.\mu=0.5 ; n_{e 0}=1.1 \times 10^{30} ; u_{0}=0.01 ; \alpha=5 / 3 ; \gamma=4 / 3\right)$.

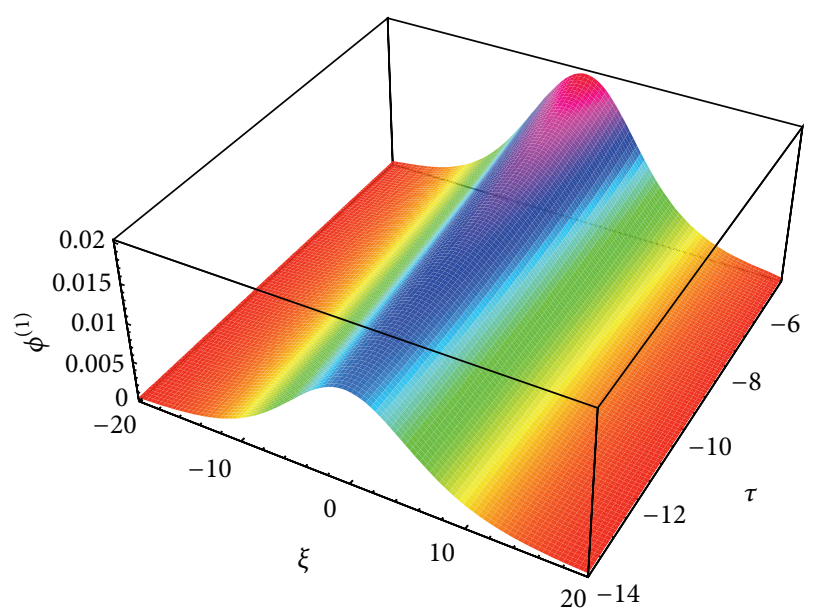

FIGURE 13: Effects of cylindrical geometry on IA K-dV solitons when both electron and ion are nonrelativistic degenerate $\left(\nu=1 ; u_{0}=\right.$ $\left.0.01 ; n_{e 0}=1.1 \times 10^{30} ; \alpha=\gamma=5 / 3\right)$.

\section{Discussion}

We have studied the nonlinear propagation of IA and mIA solitary waves in an unmagnetized, collisionless dense plasma (containing both nonrelativistic and ultrarelativistic degenerate electrons, nonrelativistic degenerate ions, and arbitrarily charged static heavy ions). The degenerate electron and ion number densities and arbitrarily charged static heavy ions significantly modify the basic properties of the solitary waves. It is also found that the amplitude of the solitary waves has been modified by the term $\mu$. In our numerical analysis, we have tried to give the idea of the variation among nonrelativistic and ultrarelativistic degenerate plasma pressure, IA and mIA solitary structures in case of planar and nonplanar geometry which makes our present work significant to understand the localized electrostatic disturbances in many

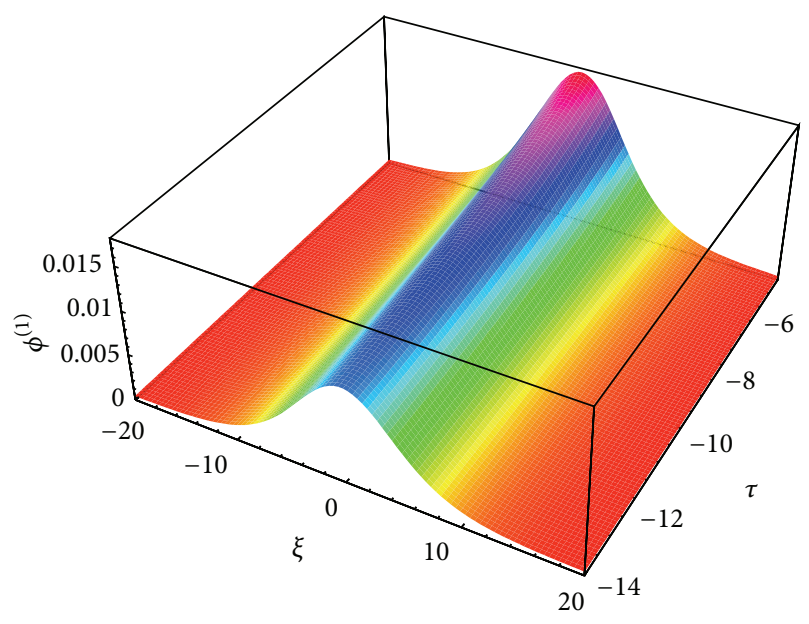

FIGURE 14: Effects of cylindrical geometry on IA K-dV solitons when ion is nonrelativistic degenerate and electron is ultrarelativistic degenerate $\left(\nu=1 ; u_{0}=0.01 ; n_{e 0}=1.1 \times 10^{30} ; \alpha=5 / 3 ; \gamma=4 / 3\right)$.

space and astrophysical plasma environments (namely, white dwarfs, neutron stars, compact planets like massive Jupiter, other exotic dense stars, and black holes). The relativistic effects of the compact objects such as white dwarf stars make them gravitationally unstable for masses larger than about 1.4 solar masses [31] and formation of neutron stars begins. This investigation would be useful to study the effects of degenerate pressure in interstellar and space plasmas [32], particularly in stellar polytropes [33], hadronic matter and quark-gluon plasma [34], protoneutron stars [35], darkmatter halos [36], and so forth. Our present investigation is a theoretical work which is applicable for matter under extreme conditions, for example, IA and mIA solitary waves propagation in the interior of compact objects $[1,2]$ where the planar and nonplanar geometry, arbitrarily charged static heavy ions, inertial ions, and degenerate plasma pressure are taken into account. This theory could be rigorously important for global nonlinear models of astrophysical compact objects.

\section{Conflict of Interests}

The authors declare that there is no conflict of interests regarding the publication of this paper.

\section{References}

[1] S. L. Shapiro and S. A. Teukolsky, Black Holes, White Dwarfs and Neutron Stars: The Physics of Compact Objects, John Wiley \& Sons, New York, NY, USA, 1983.

[2] A. K. Harding and D. Lai, "Physics of strongly magnetized neutron stars," Reports on Progress in Physics, vol. 69, no. 9, p. 2631,2006

[3] M. Temerin, K. Cerny, W. Lotko, and F. S. Mozer, "Observations of double layers and solitary waves in the auroral plasma," Physical Review Letters, vol. 48, no. 17, pp. 1175-1179, 1982.

[4] R. Boström, G. Gustafsson, B. Holback, G. Holmgren, H. Koskinen, and P. Kintner, "Characteristics of solitary waves and 
weak double layers in the magnetospheric plasma," Physical Review Letters, vol. 61, p. 82, 1988.

[5] P. O. Dovner, A. I. Eriksson, R. Bostrom, and B. Holback, "Freja multiprobe observations of electrostatic solitary structures," Geophysical Research Letters, vol. 21, no. 17, pp. 1827-1830, 1994.

[6] H. Ikezi, R. J. Tailor, and D. R. Baker, "Formation and interaction of ion-acoustic solitions," Physical Review Letters, vol. 25, article 11, 1970.

[7] H. Washimi and T. Taniuti, "Propagation of ion-acoustic solitary waves of small amplitude," Physical Review Letters, vol. 17, no. 19, pp. 996-998, 1966.

[8] N. Roy, S. Tasnim, and A. A. Mamun, "Solitary waves and double layers in an ultra-relativistic degenerate dusty electronpositron-ion plasma," Physics of Plasmas, vol. 19, no. 3, Article ID 033705, 2012.

[9] L. Nahar, M. S. Zobaer, N. Roy, and A. A. Mamun, "Ion-acoustic $\mathrm{K}-\mathrm{dV}$ and $\mathrm{mK}-\mathrm{dV}$ solitons in a degenerate electron-ion dense plasma," Physics of Plasmas, vol. 20, Article ID 022304, 2013.

[10] G. B. van Albada, "On the origin of the heavy elements," Astrophysical Journal, vol. 105, p. 393, 1947.

[11] S. Chandrasekhar, "The density of white Dwarf stars," Philosophical Magazine, vol. 11, pp. 592-596, 1931.

[12] S. Chandrasekhar, "Stellar configurations with degenerate cores," Monthly Notices of the Royal Astronomical Society, vol. 95, p. 676, 1935.

[13] M. R. Hossen, L. Nahar, and A. A. Mamun, "Roles of arbitrarily charged heavy ions and degenerate plasma pressure in cylindrical and spherical IA shock waves," Physica Scripta, vol. 89, no. 10, Article ID 105603, 2014.

[14] A. A. Mamun and P. K. Shukla, "Solitary waves in an ultrarelativistic degenerate dense plasma," Physics of Plasmas, vol. 17, no. 10, Article ID 104504, 2010.

[15] M. S. Zobaer, N. Roy, and A. A. Mamun, "DIA solitary and shock waves in dusty multi-ion dense plasma with arbitrary charged dust," Journal of Modern Physics, vol. 3, no. 8, pp. 755761, 2012.

[16] M. S. Zobaer, N. Roy, and A. A. Mamun, "Nonlinear propagation of dust ion-acoustic waves in dusty multi-ion dense plasma," Astrophysics and Space Science, vol. 343, no. 2, pp. 675681, 2013.

[17] M. M. Hossain, A. A. Mamun, and K. S. Ashrafi, "Cylindrical and spherical dust ion-acoustic Gardner solitons in a quantum plasma," Physics of Plasmas, vol. 18, no. 10, Article ID 103704, 2011.

[18] S. Chandra, S. N. Paul, and B. Ghosh, "Electron-acoustic solitary waves in a relativistically degenerate quantum plasma with twotemperature electrons," Astrophysics and Space Science, vol. 343, no. 1, pp. 213-219, 2013.

[19] M. Akbari-Moghanjoughi, "Nonlinear ion waves in FermiDirac pair plasmas," Physics of Plasmas, vol. 18, Article ID 012701, 2011.

[20] S. Chandra and B. Ghosh, "Modulational instability of electronacoustic waves in relativistically degenerate quantum plasma," Astrophysics and Space Science, vol. 342, no. 2, pp. 417-424, 2012.

[21] M. Akbari-Moghanjoughi, "Comment on "the effects of Bohm potential on ion-acoustic solitary waves interaction in a nonplanar quantum plasma" [Phys. Plasmas 17, 082307 (2010)]," Physics of Plasmas, vol. 17, no. 11, Article ID 114701, 2010.

[22] G. Manfredi, "How to model quantum plasmas," Fields Institute Communications, vol. 46, pp. 263-287, 2005.
[23] G. Brodin and M. Marklund, "Spin magnetohydrodynamics," New Journal of Physics, vol. 9, article 277, 2007.

[24] W. F. El-Taibany, A. A. Mamun, and K. H. El-Shorbagy, "Nonlinear electromagnetic perturbations in a degenerate electronpositron plasma," Advances in Space Research, vol. 50, no. 1, pp. 101-107, 2012.

[25] J. T. Mendonca and A. Serbeto, "Volkov solutions for relativistic quantum plasmas," Physical Review E, vol. 83, Article ID 026406, 2011.

[26] W. Masood and B. Eliasson, "Electrostatic solitary waves in a quantum plasma with relativistically degenerate electrons," Physics of Plasmas, vol. 18, no. 3, Article ID 034503, 2011.

[27] P. K. Shukla, A. A. Mamun, and D. A. Mendis, "Nonlinear ion modes in a dense plasma with strongly coupled ions and degenerate electron fluids," Physical Review E, vol. 84, Article ID 026405, 2011.

[28] I. Zeba, W. M. Moslem, and P. K. Shukla, "Ion solitary pulses in warm plasmas with ultrarelativistic degenerate electrons and positrons," Astrophysical Journal, vol. 750, no. 1, article 72, 2012.

[29] M. S. Zobaer, N. Roy, and A. A. Mamun, "Ion-acoustic shock waves in a degenerate dense plasma," Journal of Plasma Physics, vol. 79, no. 1, pp. 65-68, 2013.

[30] S. Maxon and J. Viecelli, "Spherical Solitons," Physical Review Letters, vol. 32, no. 1, pp. 4-6, 1974.

[31] B. Eliasson and P. K. Shukla, "The formation of electrostatic shocks in quantum plasmas with relativistically degenerate electrons," Europhysics letters, vol. 97, p. 15001, 2011.

[32] F. Ferro, A. Lavagno, and P. Quarati, "Non-extensive resonant reaction rates in astrophysical plasmas," European Physical Journal A, vol. 21, no. 3, pp. 529-534, 2004.

[33] A. R. Plastino and A. Plastino, "Stellar polytropes and Tsallis' entropy," Physics Letters A, vol. 174, no. 5-6, pp. 384-386, 1993.

[34] G. Gervino, A. Lavagno, and D. Pigato, "Nonextensive statistical effects in the quark-gluon plasma formation at relativistic heavy-ion collisions energies," Central European Journal of Physics, vol. 10, no. 3, pp. 594-601, 2012.

[35] A. Lavagno and D. Pigato, "Nonextensive statistical effects in protoneutron stars," European Physical Journal A, vol. 47, no. 4, article 52, 2011.

[36] C. Féron and J. Hjorth, "Simulated dark-matter halos as a test of nonextensive statistical mechanics," Physical Review E, vol. 77, Article ID 022106, 2008. 



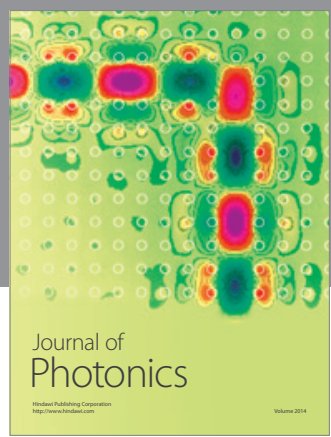

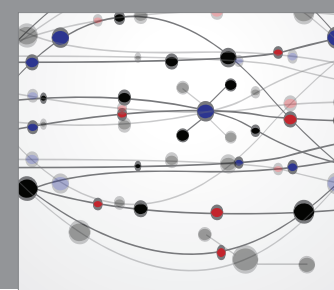

The Scientific World Journal

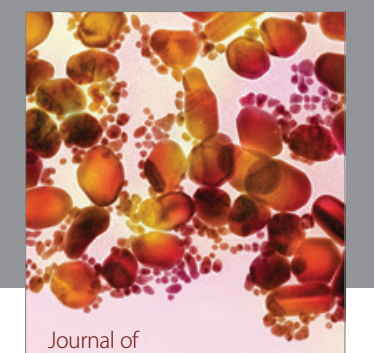

Soft Matter
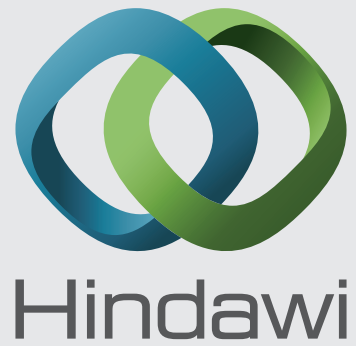

Submit your manuscripts at

http://www.hindawi.com
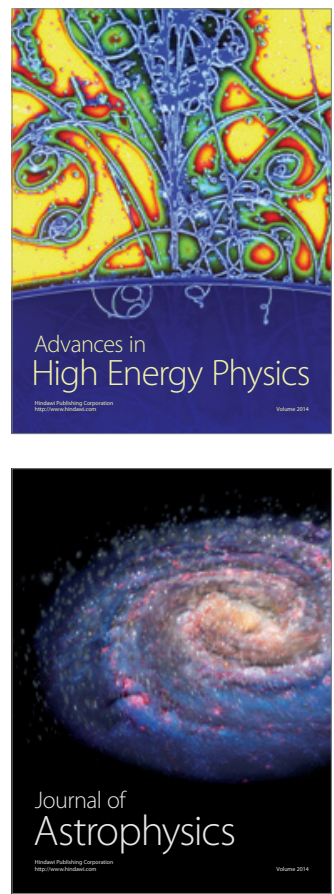
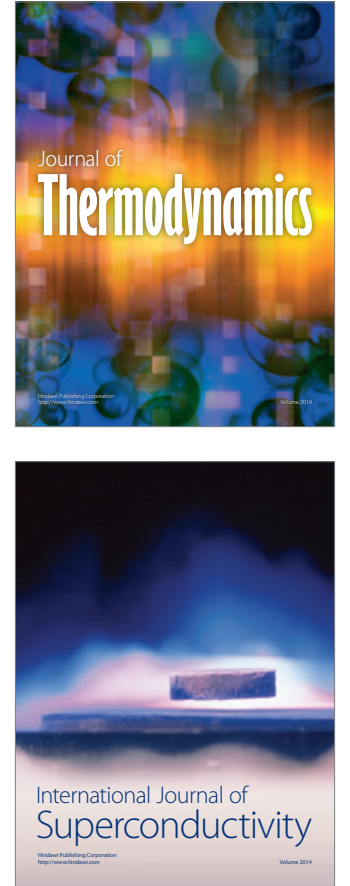
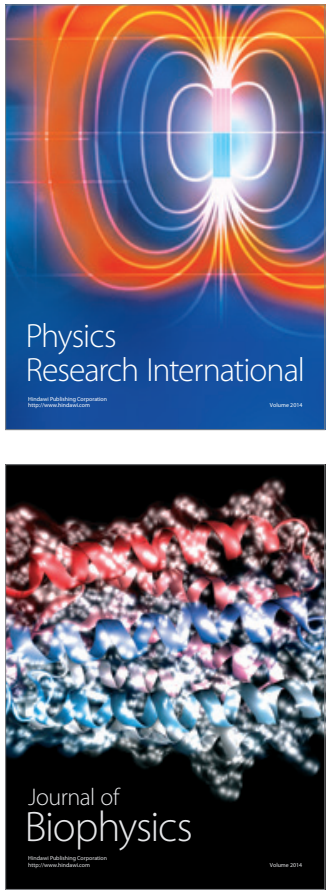
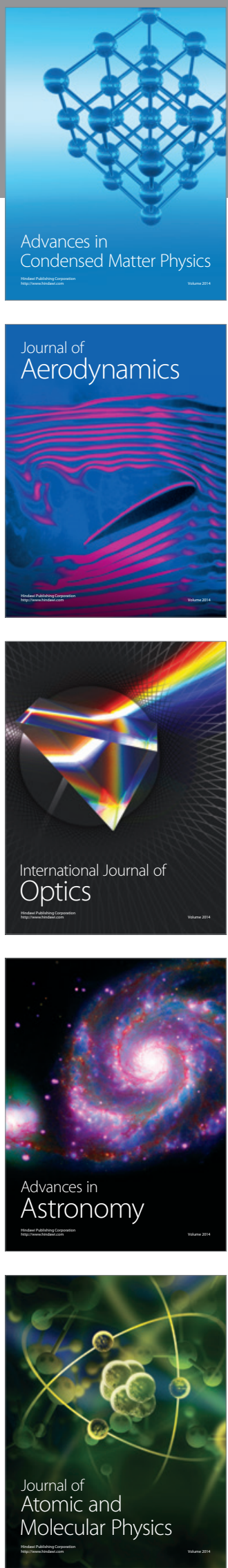